\title{
NC-Rings and Some Commutativity Conditions
}

\author{
S. Khalid Nauman, Najat M. Muthana \\ Department of Mathematics, King Abdulaziz University, Jeddah, KSA \\ Email: snauman@kau.edu.sa,nmuthana@kau.edu.sa
}

How to cite this paper: Nauman, S.K. and Muthana, N.M. (2019) NC-Rings and Some Commutativity Conditions. Advances in Pure Mathematics, 9, 143-163. https://doi.org/10.4236/apm.2019.92008

Received: January 31, 2019

Accepted: February 25, 2019

Published: February 28, 2019

Copyright $\odot 2019$ by author(s) and Scientific Research Publishing Inc. This work is licensed under the Creative Commons Attribution International License (CC BY 4.0).

http://creativecommons.org/licenses/by/4.0/

\begin{abstract}
Sum of two nilpotent elements in a ring may not be nilpotent in general, but for commutative rings this sum is nilpotent. In between commutative and non-commutative rings there are several types of rings in which this property holds. For instance, reduced, NI, AI (or IFP), 2-primal, reversible and symmetric, etc. We may term these types of rings as nearby commutative rings (in short NC-rings). In this work we have studied properties and various characterizations of such rings as well as rngs. As applications, we have investigated some commutativity conditions by involving semi-projective-Morita-contexts and right $C_{k}$-Goldie rings.
\end{abstract}

\section{Keywords}

NC-Rings, NC-Rngs, Semi-Projective-Morita-Contexts, Right $\mathrm{C}_{\mathrm{k}}$-Goldie Rings

\section{Introduction}

This expository work deals with the rings which are near to commutative rings. We know that sum of two nilpotent elements in a ring may not be nilpotent in general, for instance, for any ring $A$ with 1 , in $M_{2}(A), E_{12}$ and $E_{21}$ are nilpotent but $E_{12}+E_{21}$ is a unit. If a ring is commutative the sum of nilpotent elements is always nilpotent. In between commutative and non-commutative rings there are several types of rings in which this property holds. For instance, reduced, NI, AI (or IFP), 2-primal, reversible, and symmetric, etc. We may term these types of rings as nearby commutative rings (in short NC-rings).

In this note we will use both types of rings, we mean, associative rings that may or may not possess the multiplicative identity, and its subclass, the associative rings that possesses the multiplicative identity. For convenience, in this note, the rings of the bigger class are denoted by " $\mathbf{r} \mathbf{n} \mathbf{g} \mathbf{s}$ " (note that " $\mathrm{i}$ " is missing) while that of the subclass by " $\mathbf{r} \mathbf{i} \mathbf{g} \mathbf{s}$ ". In order to avoid unnecessary lengthy 
sentences we will not use the synonyms like "rings without identity" and "rings with identity", etc. Modules over rings are considered to be unital. Note that, the terminology "rngs" was used by Jacobson for the categories of rings without one in [1].

As applications, in the last section, we have investigated some commutativity conditions by involving semi-projective-Morita-contexts and right $C_{k}$ Goldie-rings. Though, derivations are also used and studied in several articles, we have avoided to touch them here to keep the paper of moderate length. Our focus is on NC-rings that we have studied in the second section and have discussed some properties along with examples and counter examples. We have recalled some properties of reduced, AI, NI, reversible, and symmetric rings and have proved them for rngs. It is noticed that symmetric rngs do not satisfy the initial conditions as originally defined by Lambek for rings in [2]. So, they are bifurcated as left and right symmetric rngs. We have also studied those rings which are not under the class of NC-rings, but with certain conditions, they become such rings. For instance, NCI, near-AI, quasi-AI, Armendariz, or weak-Armendariz, etc. They become NC-rings, when they are von Neumann regular. First section as usual is for preliminaries.

For basic definitions, terms and notations in ring theory we have followed the texts [1] [3]. For Morita contexts, in addition to these texts we refer [4], and in particular, for projective-Morita-contexts and semi-projective-Morita-contexts one may look at [5] [6] [7].

\section{Preliminaries}

Unless otherwise stated, we assume throughout that the lower case letters $x, x^{\prime}$ or $x_{i}$ are elements of the set denoted by the upper case letter $X$.

An element a (respt. a subset $X$ ) of a $\operatorname{rng} A$ is called nilpotent if for some natural number $n, a^{n}=0$ (respt. $X^{n}=0$ ). The least positive integer $n$ for which $a^{n}=0$ is called the index of nilpotency. The nilpotency index of a $\operatorname{rng} A$ is defined to be the supremum (possibly infinite) of the nilpotency indices of all nilpotent elements of $A$. An ideal or a subset $X \subseteq A$ is called nil if every element of $X$ is nilpotent.

We will denote by $N(A)$ the set of all nilpotent elements of $A$, by $N^{*}(A)$ the sum of all nil ideals (the so-called upper nil radical) of $A$, by $N_{*}(A)$ the intersection of all prime ideals (the so called prime or lower nil radical) of $A$, and by $N_{0}(A)$ the sum of all nilpotent ideals of $A$. It is clear that

$$
N_{*}(A) \subseteq N^{*}(A) \subseteq N(A)
$$

In case of rings with finite chain conditions on ideals, for instance for Noetherian and Artinian rngs, it is well known that

$$
N_{*}(A)=N^{*}(A)=N(A)=N_{0}(A) .
$$

A rng $A$ is called nil-simple if $N^{*}(A)=0$.

A domain is a rng which has no non-zero left or right zero divisors and an 
integral domain is a commutative ring without non-zero zero divisors.

An additive abelian group is called a zero-rng in case the product of each pair of its elements is zero. This rng is clearly commutative and its index of nilpotency is two.

A rng $A$ is prime in case for any pair of elements, $a$ and $a^{\prime}$ of $A, a A a^{\prime}=0$, then either $a=0$ or $a^{\prime}=0$, and semiprime if for any element $a$ of $A, a A a=0$ implies $a=0 . A$ is semiprime if and only if $N^{*}(A)=0$. The $\operatorname{rng} A$ is called reduced if it has no non-zero nilpotent elements, or equivalently $N(A)=0$.

A rng $A$ is von Neumann regular (in short vNR) if $\forall a \in A$, there exists $x \in A$, such that $a x a=a$ and strongly regular (in short SR) if $\forall a \in A$, there exists $x \in A$, such that $a^{2} x=a$.

For any subset $X$ of a rng $A$ the right annihilator of $X$ in $A$ is a right ideal of $A$ which is defined and denoted by:

$$
r_{A}(X)=\{a \in A \mid x a=0 \forall x \in X\} .
$$

The left annihilator $l_{A}(X)$ is defined analogously and it is a left ideal of $A$.

For singleton subsets $X=\{a\}$, we will use the notation $r_{A}(a)$ and $l_{A}(a)$ for right and left annihilators of $a$, respectively.

A right ideal $I$ of a $\operatorname{rng} A$ is said to be essential if $J$ is any other right ideal of $A$ and if

$$
I \cup J=0 \text { then } J=0 .
$$

For $I$ to be right essential, we will use the notation $I \leq_{e} A_{A}$.

It is clear that a right ideal $I$ is essential if and only if for every $x \in A$, there exists some $y \in A$ such that $0 \neq x y \in I$.

An element $a \in A$ is right singular if $r_{A}(a)$ is an essential right ideal of $A$. The set of all right singular elements is denoted by $\mathbf{z}\left(A_{A}\right)$. This is also a right ideal of $A$. The left and two-sided counter parts of these terms are defined analogously. In general, for any $\operatorname{rng} A, \mathbf{z}\left(A_{A}\right) \neq \mathbf{Z}\left({ }_{A} A\right)$ (see Example 7.6 (4) [3]). The rng $A$ is called singular if $A=\mathbf{z}(A)$ and nonsingular if $\mathbf{z}(A)=0$. Hence $A / \mathbf{z}(A)$ is nonsingular. If $A$ is a ring, then $A$ cannot be singular. Indeed, in a ring $1_{A}$ can never be a singular element.

Clearly, every reduced rng is semiprime and every commutative semiprime rng is reduced. A prime rng is also semiprime. Division rings and domains are both semiprime and reduced, while a prime rng, which is also reduced, is a domain. It is to be noted in general that $N(A)$ need not be an ideal while $N^{*}(A)$ is an ideal and a subset of $N(A)$.

A rng $A$ is termed as 2-primal in [8] if its prime radical (the intersection of all prime ideals) is $N(A)$ and SP I in [9] if for every element $a$ of $A$, the factor ring $A / r_{A}(a A)$ is 2-primal.

Further, a rng is an $N I$-rng, as defined in [10] if $N^{*}(A)=N(A)$ and an $N C I$-rng in [11] if $N(A)$ has a non-zero ideal.

Clearly, every reduced ring is NI as in this case $N(R)=0$, which is of course, an ideal of $A$. 
A rng $A$ is called an IFP rng [12] (in our term an $A I-r n g$ ) in case for any pair of elements $a, b$ of $A$, if $a b=0$, then $a A b=0$. Near-IFP (or Near-AI) rngs are introduced in [13] and are characterized in Proposition.1.2. ([13]) as $A$ is a near-AI rng if and only if for a non-zero nilpotent element a of $A, A a A$ contains a non-zero nilpotent ideal, while almost simultaneously, but seems to be independently, quasi-IFP (in our terms quasi-AI) rngs are introduced in [14].

By definitions, every reduced rng is an AI-rng, an AI-rng is quasi-AI, a quasi-AI rng is near-AI. By a simple argument it can be deduced that the converse of above statement holds if the ring is semiprime (see [13]; Prop.1.2, Prop. 1.5).

If a ring $A$ is of bounded index of nilpotency, then $A$ is reduced if and only if $A$ is NI (or NCI) and semiprime ([11]; Prop. 1.3).

A rng $A$ is called abelian if its idempotents are central and $A$ is termed as left (respt. right) duo if every left (respt. right) ideal is also an ideal. It is proved in ([11]; Prop. 1.4) that if $A$ is a vNR ring, then the properties of being reduced, left duo, right duo, abelian, NI, NCI and 2-primal are all equivalent. Similarly, it is proved in ([13]; Prop.1.6), that if $A$ is a vNR ring, then the properties of being reduced, left (or right) duo, abelian, NI, AI, and near-AI are all equivalent. Hence, one may deduce that on a vNR ring all these different types of rings are equivalent (see Theorem 4.3.6).

We assume that the datum

$$
K(A, B)=\left[A, B, M, N,\langle,\rangle_{A},\langle,\rangle_{B}\right]
$$

is a Morita context (in short, " $m c$ ") in which $A$ and $B$ are rngs, $M$ and $N$ are $(B, A)$ - and $(A, B)$-bimodules, respectively,

$$
\langle,\rangle_{A}: N \otimes_{B} M \rightarrow A \text { and }\langle,\rangle_{B}: M \otimes_{A} N \rightarrow B
$$

are bimodule morphisms with the associativity (or compatibility) conditions

$$
\text { 1) } m^{\prime}\langle n, m\rangle_{A}=\left\langle m^{\prime}, n\right\rangle_{B} m \text { and 2) }\langle n, m\rangle_{A} n^{\prime}=n\left\langle m, n^{\prime}\right\rangle_{B}
$$

where $\langle,\rangle_{A}$ and $\langle,\rangle_{B}$ are the Morita maps (in short, mc maps). The images $I=\langle N, M\rangle_{A}$ and $J=\langle M, N\rangle_{B}$ are the trace ideals of $A$ and $B$, respectively.

A Morita context $K(A, B)$ is said to be a "projective Morita context", in short a "pmc" (or strict), if both mc maps, $\langle,\rangle_{A}$ and $\langle,\rangle_{B}$, are epimorphisms. $K(A, B)$ is said to be a "semi-projective Morita context", or a "semi-pmc", if one of the mc maps, $\langle,\rangle_{A}$ or $\langle,\rangle_{B}$, is an epimorphism (see [5] [6] [7] for further details).

Let $A$ and $B$ be rings. In case an mc $K(A, B)$ of rings is a pmc, i.e., if both mc maps $\langle,\rangle_{A}$ and $\langle,\rangle_{B}$ are epimorphisms, then they become isomorphisms. In this case, the category of right (respt. left) $A$-modules is equivalent to the category of right (respt. left) $B$-modules i.e.,

$$
\operatorname{Mod}-A \approx \operatorname{Mod}-B(A-M o d \approx B-M o d)
$$

and moreover,

$$
\operatorname{Cent}(A) \cong \operatorname{Cent}(B)
$$


If $K(A, B)$ is a pmc of rings, then the rings $A$ and $B$ are said to be Morita similar (or Morita equivalent). Common properties shared by Morita similar rings are termed as Morita invariant. For instance, being prime or semiprime are Morita invariant, while being reduced, commutative, domain, division rings or fields are not Morita invariant.

\section{NC-Rngs and Their Relations}

In this section we will introduce nearby commutative rngs (in short NC-rngs). We will investigate some properties of NC-rngs and demonstrate some examples and counter examples. Then we will discuss and expose some of those rngs which satisfy the condition of NC-rngs. For instance, reduced, AI, reversible and symmetric rngs.

For rings following sequence is given in ([9]; Fig.1, Various types of 2-primal rings):

Reduced $\subseteq$ Symmetric $\subseteq$ Reversible $\subseteq \mathrm{AI} \subseteq \mathrm{PS} \mathrm{I} \subseteq 2$-Primal $\subseteq$ NI-ring

In fact, symmetric rings are reversible, but a reversible ring may not be symmetric [9] [15]. In case of rngs a symmetric rng may not be reversible also (see Example 3.4.7 (2)). Note that, the definition of a symmetric ring, as introduced by Lambek [2], does not justify its property for a symmetric rng. Thus we need to bifurcate it into two parts, right symmetric rng and left symmetric rng (see [16] for details). We have given examples that they are different rngs. They become identical at least if the rng is commutative or if it is a ring. Thus we have the following extended sequences for rngs which are irreversible in general (see counter examples in [9] [10] [15] [16]).

$$
\begin{aligned}
& \subseteq \text { Right Symmetric } \subseteq \\
\text { Reduced } \subseteq \text { Symmetric } & \subseteq \text { Reversible } \subseteq \mathrm{AI} \subseteq \text { PS I } \subseteq 2 \text {-Primal } \subseteq \mathrm{NI}-\text { rng } \\
& \subseteq \text { Left Symmetric } \subseteq
\end{aligned}
$$

\subsection{NC-Rngs}

Definition 3.1.1. A rng $A$ is nearby commutative, in short an NC-rng, in case the sum of any two nilpotent elements of $A$ is nilpotent.

Proposition 3.1.2. $A$ rng $A$ is $N C$ if and only if $N(A)$ is an additive subgroup of $A$.

Proof: One way is obvious. Assume that $A$ is an NC-rng. Clearly, $0 \in N(A)$, and if $x \in N(A)$, then so is $-x \in N(A) \Rightarrow \forall x, y \in N(A), \quad x-y \in N(A)$.

Examples 3.1.3. Field, division rings, domains, reduced, symmetric, reversible, 2-primal, AI, and NI-rngs are NC-rngs. The smallest class of non-commutative NC-rngs is the class of reduced rngs. Conversely, Example 3.1.6 below shows that an NC-rng properly subsumes all above mentioned classes of rngs.

Hence, extending sequences (2) we have following irreversible sequences of rngs:

$$
\subseteq \text { Right Symmetric } \subseteq
$$


Reduced $\subseteq$ Symmetric $\subseteq$ Reversible $\subseteq \mathrm{AI} \subseteq \mathrm{PS} \mathrm{I} \subseteq 2$-Primal $\subseteq \mathrm{NI} \subseteq \mathrm{NC}$-rng (3)

$$
\subseteq \text { Left Symmetric } \subseteq
$$

Proposition 3.1.4. Being "NC-rings" is not a Morita invariant property.

Proof: For any ring $A$, it is known that $A$ and $M_{n}(A)$ are Morita similar. Let $A$ be an NC-ring. But $M_{n}(A)$ is not an $N C$ ring. Because, $E_{n 1}, E_{1 n} \forall n \geq 2$ are nilpotent but the sum $E_{n 1}+E_{1 n}$ is not nilpotent. Hence, for a ring, being NC is not Morita invariant.

Example 3.1.5. (NC-rngs which are also NI-rngs).

1) Consider the non-commutative ring of order $4, V=\{0, a, b, a+b\}$ with $\operatorname{Char}(V)=2$ and the elements satisfy the relations

$$
a^{2}=a b=a ; b^{2}=b a=b .
$$

See also Remarks 3.4.8. and Examples 3.4.10. This is a rng, called the Klein 4 -rng or right absorbing ring in [16]. Its only non-zero nilpotent element is $a+b$ and the only nilpotent ideal is $\{0, a+b\}$. Hence $V$ is an NC-rng as well as an NI-rng.

2) Set $R=Z_{2} \times V=\{0,1, a, b, 1+a, 1+b, a+b, 1+a+b\}$ assume that the relations in $V$ also hold in $R$. Again, $\{0, a+b\}$ is the only nilpotent ideal of $R$. Hence $R$ is an NC-ring as well as an NI-ring.

Example 3.1.6. A ring which is an NC-ring but not an NI-ring.

Let $G$ be a free semigroup generated by $\left\{x_{i}: i \in I\right\}$ and $R$ any NI-ring. Then the group ring $R G$ is the free $R$-ring, $R G:=R\left\langle x_{i} \in I\right\rangle$. Then $U(R G)=U(R)$. Let the set of nilpotent elements be $N(R G)$. Assume that

$(R G \backslash R) \cap N R(G) \neq \Phi$. If $f$ is some arbitrary nilpotent element in $R G \backslash R$, then $1-f$ must be a unit. So $1-f \in U(R)$ must be a constant. This means that $f$ is a constant polynomial. It is a contradiction. Hence $N R(G)=0$ or consists of only elements of $R$. But $R$ is an NI-ring, this implies that $N R(G)=N(R)$. Finally, $N(R)$ is not an ideal in $R G$. Hence $R G$ is neither NI nor NCI, but it is clear that sum of two nilpotent elements in $R G$ is nilpotent, so it is an NC-ring.

Proposition 3.1.7. In a ring $A$, if $U(A)$ is abelian, then $A$ is an $N C$-ring.

Proof: Let $x$ and $y$ be nilpotent elements of the ring $A$. Then $u=1-x$ and $v=1-y$ are units. But

$$
u v=(1-x)(1-y)=1-x-y+x y
$$

and

$$
v u=(1-y)(1-x)=1-y-x+y x
$$

So,

$$
u v-v u=x y-y x=0 .
$$

This means that all nilpotent elements commute with each other. In particular, if $m$, and $n$ are indices of nilpotencies of $x$ and $y$, respectively, then $(x+y)^{m+n}=0$.

Examples 3.1.8. The converse of above theorem does not hold in general. For instance, in the integral ring of quaternions, $\mathbf{Z}\left(Q_{8}\right)$, only the nilpotent element 
is 0 , which commutes with everything, but the group of units in $\mathbf{Z}\left(Q_{8}\right)$ is non-abelian. Let $G$ be a free semigroup generated by $\left\{x_{i}: i \in I\right\}$ and $R$ any commutative ring. Then the group ring $R G$ is the free $R$-ring $:=R\left\langle x_{i} \in I\right\rangle$. As in Example 3.1.6. above, $U(R G)=U(R)$ and the set of nilpotent elements $N(R G)=N(R)$. Since, in general, $\left\{x_{i}: i \in I\right\}$ are noncommutative, $R G$ is noncommutative. But $U(R)$ and simultaneously $N(R)$ are commutative.

Let us define that $U N(A):=\{u: u=1-x$, with $x \in N(A)\}$. We prove the following interesting result.

Proposition 3.1.8. If $A$ is an NC-ring with the property that $N(A)$ is multiplicatively closed, then $U N(A)$ is a normal subgroup of $U(A)$.

Proof: Because $1 \in U N(A)$, it is non-empty. Let $u, v \in U N(A)$. Then there exist $x, y \in N(A)$ such that $u=1-x$ and $v=1-y$. Because $N(A)$ is additively and multiplicatively closed,

$$
u v=1-x-y+x y \in U N(A) .
$$

Let $n$ be the index of nilpotency of $y$. Then,

$$
v^{-1}=1+y+y^{2}+\cdots+y^{n-1},
$$

where $y+y^{2}+\cdots+y^{n-1}$ is nilpotent. This means that $v^{-1} \in U N(A)$. Finally, let $w \in U(A)$. Then $\forall u \in U N(A)$, with $u=1-x, x \in N(A)$,

$$
w u w^{-1}=w(1-x) w^{-1}=1-w x w^{-1} .
$$

But clearly, $w x w^{-1}$ is nilpotent, $w u w^{-1} \in U N(A)$. Hence $U N(A)$ is a normal subgroup of $U(A)$.

\subsection{Reduced Rings}

Definition 3.2.1. A rng $A$ is reduced if it has no non-trivial nilpotent element, equivalently $N(A)=0$ or that index of nilpotency of $A$ is one.

Because a reduced ring has no non-trivial nilpotent elements, it is an $N C$-rng.

Examples 3.2.2. 1) Fields, division rings, integral domains are reduced rings. A domain is a reduced rng. A zero-rng $D(\neq 0)$ is not reduced.

2) Let $A$ be a rng. Let $M_{n}(A)$ be the set of all $n \times n$-matrices over $A$. Then for $n \geq 2, M_{n}(A)$ is not reduced as $M_{n}(A)$ admits nilpotent elements of higher indices than one. Its subrng, the set of upper triangular matrices $U T M_{n}(A)$ is also not reduced. Clearly, in $U_{T M}(A)$ the matrix $a E_{12}, \forall a \in A$ is nilpotent of index two. Above arguments also show that property of being reduced is not Morita invariant.

In the following proposition the results in literature are available for rings. We verify that these are also hold for rngs.

Propositions 3.2.3.

1) Reduced rngs are semiprime and a commutative semiprime rng is reduced.

2) SR rngs are reduced, hence commutative. vNR rngs are also reduced.

3) Reduced rngs are nonsingular and a commutative nonsingular rng is reduced.

4) If $A$ is a nonsingular rng and $N(A) \subseteq \operatorname{Cent}(A)$, then $A$ is reduced. 
Proof: 1) Obviously, reduced rings have no non-zero nilpotent ideals, so they are semiprime. Conversely, assume that

$$
a^{2}=0 \Rightarrow \forall x \in A, \text { axa }=0 \Rightarrow a=0 .
$$

2) Indeed, if $A$ is an SR rng and $a \in A$ is nilpotent with index $n$, then there exists $x \in A$ such that

$$
a=a^{2} x=a a^{2} x=\cdots=a a^{n-1} x=0 .
$$

Hence $A$ is reduced.

3) Let $A$ be a reduced rng and $0 \neq a \in \mathbf{Z}\left(A_{A}\right)$. Then $r_{A}(a) \leq_{e} A_{A}$. If $b \in a A \cap r_{A}(a)$, then $b=a x$ for some $x \in A$ and $a b=0$. But

$$
(b a)^{2}=0 \Rightarrow b a=0 \Rightarrow b^{2}=b a x=0 \Rightarrow b=0 .
$$

Hence $\mathbf{z}\left(A_{A}\right)=0$ and similarly $\mathbf{z}\left({ }_{A} A\right)=0$. Hence $A$ is nonsingular.

Conversely, assume that $0 \neq a \in A$, such that

$$
a^{2}=0 \Rightarrow a \in r_{A}(a) \text {. }
$$

Let $I \neq 0$ be an ideal such that $r_{A}(a) \cap I=0$. Then for any $y \in I$,

$$
y a \in r_{A}(a) \cap I \Rightarrow y a=0 \Rightarrow I \subseteq r_{A}(a) \Rightarrow a=0 .
$$

Hence $A$ is reduced.

4) First we prove that every nilpotent element that lies in the center is singular. Let $a \in A$ be a nilpotent element of index $n$ that lies in the center of $A$. Then $a^{n-1} \in r_{A}(a)$. Now for any $x \in A$, because $r_{A}(a)$ is a right ideal, $a^{n-1} x \in r_{A}(a)$. Because $a$ is a central element, $x a^{n-1} \in r_{A}(a)$. This means that $r_{A}(a) \leq_{e} A_{A}$, hence $a \in \mathbf{Z}\left(A_{A}\right)$. By similar arguments we conclude that $a \in \mathbf{Z}\left({ }_{A} A\right)$ and so $a \in \mathbf{Z}(A)$.

By contraposition if

$$
x \in N(A) \subseteq \operatorname{Cent}(A),
$$

then $x$ must be singular. But $A$ is nonsingular, then $x$ must be 0 . Hence $A$ is reduced.

\subsection{AI-Rngs and Generalizations}

AI-rngs have many names in literature. H.E. Bell in [12] called an ideal $I$ of $A$ to be with the insertion-of-factors-property in case for any pair of elements $a, a^{\prime}$ of $A, a a^{\prime} \in I$ implies that $a A a^{\prime} \subseteq I$. Thus any rng with the property that for $a a^{\prime}=0 \Rightarrow a A a^{\prime}=0$ is popular as an IFP-rng, the short form of insertion of factors property. Marks in [9] called it with property (S I). He continued this term from Shin in [17]. Narbonne called it semicommutative (see reference in [13]) and many others followed this term (e.g., [18] [19]). Habeb in [20] called it zero insertive. We prefer to call it an AI-rng due to the fact that in this rng all "Annihilators", left or right, are "Ideals".

Lemma 3.3.1. For any rng $A$ the following are equivalent:

1) For any pair $a, b \in A, a b=0 \Rightarrow a r b=0, \forall r \in A$.

2) Every right annihilator $r_{A}(X)$ of $X \subseteq A$ is an ideal. 
3) Every left annihilator $l_{A}(X)$ of $X \subseteq A$ is an ideal.

4) For every $a \in A$, right annihilator $r_{A}(a)$ is an ideal.

5) For every $a \in A$, left annihilator $l_{A}(a)$ is an ideal.

6) For every $a \in A$ and $b \in A, r_{A}(a) \leq r_{A}(a b)$.

7) For every $a \in A$ and $b \in A, l_{A}(a) \leq l_{A}(b a)$.

Proof: 1) $\Leftrightarrow 2$ )

Let $S \neq \Phi$ be a subset of $A$. Then $r_{A}(S)$ is a right ideal of $A$. We prove that if 1) holds, then it is also a left ideal of $A$. Let $a \in r_{A}(S)$. So for any $b \in S$, $b a=0 \Rightarrow b r a=0, \forall r \in A \Rightarrow r a \in r_{A}(S)$. Hence 2) holds.

Now assume that 2) holds. Let for some $a, b \in A$,

$$
a b=0 \Rightarrow b \in r_{A}(a) \Rightarrow \forall r \in A, r b \in r_{A}(a) \Rightarrow a r b=0 .
$$

1) $\Leftrightarrow 4) \quad a x=0 \Leftrightarrow x \in r_{A}(a)$. But $a b x=0 \Leftrightarrow x \in r_{A}(a b)$.

The rest can analogously be proved.

Definition 3.3.2. If any one of the conditions of Lemma 3.3.1 is satisfied then the rng is called an AI-rng. If $1 \in A$, then it is an AI-ring.

It is clear from the sequences 3 ) that $\mathrm{AI}$ is an NC-rng. We pose here an alternate proof.

Proposition 3.3.3. AI-rng is an NC-rng.

Proof: Let $A$ be an $A I$-rng and $a, b \in A$ be two nilpotent elements of indices $m, n$, respectively. We want to prove that $a+b$ is nilpotent. A monomial in the expansion of $(a+b)^{t}$ is an expression of the form

$$
x=a^{r_{1}} b^{s_{1}} a^{r_{2}} b^{s_{2}} \cdots a^{r_{k}} b^{s_{k}}, \quad \text { where } r_{1}+s_{1}+\cdots+r_{k}+s_{k}=t=\max (m, n) .
$$

Now,

$$
a^{m}=0 \Rightarrow a^{m-1} a=0 \Rightarrow a^{m-1} b a=0 .
$$

Such insertions eventually make $x=0$ and so we conclude that

$$
(a+b)^{t}=\sum_{r_{i}, s_{i}=0}^{t} a^{r_{1}} b^{s_{1}} a^{r_{2}} b^{s_{2}} \cdots a^{r_{k}} b^{s_{k}}=0 .
$$

Hence $A$ is an NC-rng.

Proposition 3.3.4. A semiprime AI-rng is nonsingular.

Proof: Let $A$ be a semiprime $A I$-rng and $0 \neq x \in A$ be such that $x \in \mathbf{Z}\left(A_{A}\right)$. Then there exists $0 \neq a \in A$, such that

$$
\begin{aligned}
& a \in r_{A}(x) \cap x A \Rightarrow x a=0 \\
& \Rightarrow x A a=0 \Rightarrow a^{2}=0 \\
& \Rightarrow a A a=0 \Rightarrow a=0 .
\end{aligned}
$$

This is a contradiction that $r_{A}(x)$ is essential in $A_{A}$. We conclude that

$$
x=0 \Rightarrow \mathbf{z}\left(A_{A}\right)=0 \text {. }
$$

Similarly

$$
\mathbf{Z}\left({ }_{A} A\right)=\mathbf{Z}(A)=0 .
$$

Hence $A$ is nonsingular. 
Marks in [9] [10] [15] used the term $N I$ for those rings in which $N(A)$ is an ideal, then naturally, $N^{*}(A)=N(A)$. A ring $A$ is called left (right) duo if each left (right) ideal is an ideal.

Proposition 3.3.5. Following are true for any rng.

1) Every left (right) duo rng is an AI-rng.

2) Every reduced rng is an AI-rng.

3) Every AI-rng is an NI-rng.

4) Every idempotent in an AI-domain is central. Hence an AI-domain is abelian.

5) Every idempotent in an AI-ring is central. Hence an AI-ring is abelian.

6) For any rng $A$,

$A$ is nil-simple $\Rightarrow A$ is semiprime $\Leftarrow A$ is reduced.

If $A$ is commutative, then

$$
\text { Nil-simple }=\text { Semiprime }=\text { Reduced. }
$$

Proof: 1) Let $A$ be a left duo ring. Let for some $a, b \in A$,

$$
a b=0 \Rightarrow a \in l_{A}(b) \Rightarrow a r \in l_{A}(b) \Rightarrow a r b=0,
$$

$\forall r \in A$. Hence $A$ is an $A I$-rng.

2) Let $A$ be a reduced ring. Assume that for some pair of elements $a, b \in A$, $a b=0$. Then for any $x \in A$,

$$
(b x a)(b x a)=0 \Rightarrow b x a=0 .
$$

But then

$$
(b a)^{2}=0 \Rightarrow b a=0 \Rightarrow a x b=0 .
$$

3) If $N(A)=0$, then we are done. So let $0 \neq a \in N(A)$. Then for some natural number $n>1$, and for any $r \in A$,

$$
a^{n} r=0 \Rightarrow a^{n} r r=0 \cdots(a r)^{n}=0 .
$$

Hence ar $\in N(A)$. Similarly, $r a \in N(A)$.

Let $a, b \in N(A)$ with indices $n, m$, respectively. After some calculations one can verify that $(a-b)^{t}=0$, for some natural number $t \geq n+m$. So $N(R)$ is an additive group. Hence $N(A)$ is an ideal.

4) Let $A$ be an $A I$-domain (recall that a domain is a rng without non-zero zero-divisors). Let $e \in A$ be an idempotent. Then $\forall r \in A$,

$$
\begin{aligned}
e\left(e^{2}-e\right)=0 & \Rightarrow e r\left(e^{2}-e\right)=0, \\
& \Rightarrow \text { ere }{ }^{2}=\text { ere } \\
& \Rightarrow \text { ere }=\text { er }
\end{aligned}
$$

Similarly, ere $=$ re.

5) If $1 \in A$, then

$$
\begin{aligned}
& e^{2}-e=e(e-1)=0 \\
& \Rightarrow e r(e-1)=0 \\
& \Rightarrow \text { ere }=e r=r e .
\end{aligned}
$$

6) Holds by definitions. 
Examples 3.3.6. 1) Fields, division rings, integral domains are AI-rings and all commutative rngs, domains, zero-rngs, and reduced rngs are AI-rngs.

2) Being AI is not Morita invariant.

The $3 \times 3$ upper triangular matrix rng $U T_{3}$ may not be AI. Indeed,

$$
\left(-a E_{11}+a E_{12}\right)\left(a E_{23}\right)=0
$$

while, in general,

$$
\left(-a E_{11}+a E_{12}\right)\left(a E_{22}\right)\left(a E_{23}\right) \neq 0 .
$$

In particular, if $A$ is an AI-ring, then $U T_{3}$ is not an AI ring. (Simply, replace a by 1 in above example). Hence $M_{n}(A)$ is not an AI-ring. This shows that for any ring being $\mathrm{AI}$ is not Morita invariant.

Proposition 3.3.7. a) For an AI-rng A, the subrng of $3 \times 3$ upper triangular matrix rng,

$$
S=\left\{S_{k}=\left[\begin{array}{ccc}
a_{k} & a_{k 12} & a_{k 13} \\
0 & a_{k} & a_{k 23} \\
0 & 0 & a_{k}
\end{array}\right]: a_{k}, a_{k i j} \in A\right\}
$$

is an AI-rng. Hence, $S$ is an NC-rng.

b) If $A$ is an AI-ring, then $S$ is an AI-ring.

Proof: a) For the verification of this claim, assume that $s_{1} s_{2}=0$. Then we get the following identities

1) $a_{1} a_{2}=0$.

2) $a_{1} a_{212}+a_{112} a_{2}=0$.

3) $a_{1} a_{213}+a_{112} a_{223}+a_{113} a_{2}=0$.

4) $a_{1} a_{223}+a_{123} a_{2}=0$.

Now we claim that the product

$$
S_{1} S s_{2}=0 .
$$

For this it is enough to prove that

5) $a_{1} A a_{2}=0$.

6) $a_{1} A a_{212}+a_{1} A a_{2}+a_{112} A a_{2}=0$.

7) $a_{1} A a_{213}+a_{112} A a_{223}+a_{1} A a_{223}+a_{1} A a_{2+} a_{112} A a_{2}+a_{113} A a_{2}=0$.

8) $a_{1} A a_{223}+a_{1} A a_{2}+a_{123} A a_{2}=0$.

In above Equations (1)-(4) we apply the equivalent condition 3 of Proposition 3.2.1 of being AI repeatedly. Hence 5) holds from 1). While 6) and 8) hold from 1), 4) and 5), and finally, 7) from 3) and the remaining. (For more details, see [19]) where they considered $A$ to be reduced.

b) Clearly the $3 \times 3$ identity matrix belongs to $S$.

Example 3.3.8. An abelian ring which is not $A I$

Let $A$ be an abelian ring. Then

$$
R=\left\{\left[\begin{array}{llll}
a & b & c & d \\
0 & a & f & g \\
0 & 0 & a & k \\
0 & 0 & 0 & a
\end{array}\right]: a, b, c, d, f, g, k \in A\right\}
$$


is also abelian (see details in [21]). Then

$$
\left(E_{12}-E_{13}\right)\left(E_{24}+E_{34}\right)=0
$$

But

$$
\left(E_{12}-E_{13}\right) E_{23}\left(E_{24}+E_{34}\right)=E_{14} \neq 0
$$

Hence $R$ is not an AI-ring.

$\mathrm{NCI}-$, near-AI and quasi-AI rngs are introduced in [9] [13] and [14], respectively.

Definitions 3.3.9. Let $A$ be a rng. We define that:

1) $A$ is an NCI-rng in case $N(A)$ contains a non-zero ideal of $A$.

2) $A$ is near-AI in case $N(A)$ contains a non-zero nilpotent ideal of $A$.

3) $A$ is quasi-AI if for any nilpotent element a of $A, A a A$ is a nilpotent ideal of $A$. (Equivalently $N(A)=N_{0}(A)$ ).

For detailed studies of these classes of rngs we refer the reader to the above cited articles. It is clear by definitions that

$$
\begin{aligned}
& \mathrm{AI} \Rightarrow \mathrm{NI} \Rightarrow \mathrm{NCI} \\
& \mathrm{AI} \Rightarrow \text { near-AI } \Rightarrow \text { quasi-AI }
\end{aligned}
$$

\section{Examples 3.3.10.}

1) ([11], Example 1.2) Let $A$ be any rng. Set $R=M_{2}\left[U T M_{2}(A)\right]$. Then $R$ is not NI, but the ideal

$$
I=\left[\begin{array}{ll}
{\left[\begin{array}{ll}
0 & A \\
0 & 0
\end{array}\right]} & {\left[\begin{array}{ll}
0 & A \\
0 & 0
\end{array}\right]} \\
{\left[\begin{array}{ll}
0 & A \\
0 & 0
\end{array}\right]} & {\left[\begin{array}{ll}
0 & A \\
0 & 0
\end{array}\right]}
\end{array}\right]
$$

of $R$ is nilpotent. Hence $N I \nLeftarrow N C I$.

2) ([13], Example 1.3) Let $A$ be reduced. Set $R=U T M_{2}(A)$. Then $R$ is neither abelian nor AI, but it is near-AI.

3) Now we pose an example for near-AI $\nLeftarrow$ quasi-AI .

Let $R$ be a ring and $I \neq 0$ a nilpotent ideal of $R$ such that every element of $R \mid I$ is a unit. (For example a local ring). By Prop. 1.10 in [13], $R_{n}=M_{n}(R)$ is near-AI. Let $0 \neq\left(r_{i j}\right) \in N\left(R_{n}\right)$. Then $R_{n}\left(r_{i j}\right) R_{n}=R_{n}$ is nilpotent if $r_{i j} \in I$, otherwise it is not nilpotent in general. The ideal $R_{n} M_{n}(I) R_{n}$ of $R_{n}$ is proper and nilpotent. Hence we conclude that it is not quasi-AI.

If a ring $A$ is of bounded index of nilpotency, then $A$ is reduced if and only if $A$ is NI (or NCI) and is semiprime ([11]; Prop. 1.3).

\subsection{Reversible and Symmetric Rngs}

Definition 3.4.1. Cohn in [22] called a ring $A$ to be reversible in case whenever $a b=0 \Rightarrow b a=0, \forall a, b \in A$.

Anderson and Camillo in [23] called it a ZC2-rng.

Examples 3.4.2: 1) All commutative rngs, domains, and zero-rngs are reversible. 
2) Reduced rngs are also reversible. Indeed, if $a b=0$, then

$$
(b a)^{2}=b(a b) a=0 \Rightarrow b a=0 \text {. }
$$

3) Clearly, for any $\operatorname{ring} A, M_{n}(A)$ is not reversible. Hence the property of being reversible is not Morita invariant.

4) If $A$ is a reversible rng, then the polynomial rng $A[x]$ may not be reversible (see ([18]; Example 2) \& ([19]; Example 2.1)).

5) Example 3.1.5. (1) Clearly $\mathrm{V}$ is not reduced as $a+b$ is a non-zero nilpotent element and it is not reversible as

$$
a(a+b)=0 \neq(a+b) a=a+b .
$$

Similarly the ring in Example 3.1.5. 2) is neither reduced nor reversible.

6) Consider the group of quaternions $Q_{8}=\{ \pm 1, \pm i, \pm j, \pm k\}$, then the group algebra $\mathbf{Z}_{2}\left(Q_{8}\right)$ is a reversible ring. In fact, the elements of $\mathbf{Z}_{2}\left(Q_{8}\right)$ are the

$\mathbf{Z}_{2}$-linear combinations of the elements of the set $\left\{z_{g}: g \in Q_{8}\right\}$. It is a routine work to check that $\mathbf{Z}_{2}\left(Q_{8}\right)$ is reversible. Moreover, from Proposition 6 of [15] it is clear that $\mathbf{Z}_{2}\left(Q_{8}\right)$ is an AI-ring. Further, in ([15], Example 7), it is proved that $\mathbf{Z}_{2}\left(Q_{8}\right)$ is right duo.

Proposition 3.4.3. Reversible rngs are

1) AI-rngs and

2) $N C$-rngs.

Proof: 1) Let $A$ be reversible. Then for some $a, b \in A, a b=0 \Rightarrow b a=0$. Then $\forall r \in A,(b a) r=b(a r)=(a r) b=a r b=0$.

2) Because every AI-rng is NC, so is a reversible rng.

Proposition 3.4.4. A reversible $v N R$ ring is an $S R$ ring.

Proof: Let $A$ be a vNR ring. Then $\forall a \in A$, there exists $r \in A$, such that $a=\operatorname{ara} \Rightarrow$

$$
a(1-r a)=0 \Rightarrow(1-r a) a=0 \Rightarrow a=r a^{2}
$$

Hence, $A$ is SR.

Definition 3.4.5. J. Lambek in [2] introduced symmetric rngs: A $\operatorname{rng} A$ is symmetric if for any $a, b, c \in A, a b c=0 \Leftrightarrow a c b=0$.

Remarks 3.4.6. 1) If the $\operatorname{ring} A$ is equipped with identity, say $1_{R}$, then Lambek proved that: $A$ is a symmetric ring if and only if for any $a_{i} \in A$, where $i=1, \cdots, n$, the product

$$
\prod_{i=1}^{n} a_{i}=0 \Leftrightarrow \prod_{i=1}^{n} a_{\sigma(i)}=0, \text { where } \sigma:\{1, \cdots, n\} \rightarrow\{1, \cdots, n\}
$$

is a permutation. This characterization may not hold if $A$ does not have $1_{A}$.

2) If a $\operatorname{rng} A$ satisfies the condition

$$
\prod_{i=1}^{n} a_{i}=0 \Leftrightarrow \prod_{i=1}^{n} a_{\sigma(i)}=0, \text { where } \sigma:\{1, \cdots, n\} \rightarrow\{1, \cdots, n\},
$$

then in [23] it is called a $Z C_{n}$-rng. It is proved there that for all $n \geq 3$, if $A$ satisfies $Z C_{n}$ then $A$ also satisfies $Z C_{n+1}$. Hence, inductively, $Z C_{3}$ implies $Z C_{n}$ for all $n \geq 3$ ([23]; Theorem I.1 \& Corollary I.2). The converse does not hold in general. Moreover, $Z C_{2}$ does not imply $Z C_{3}$ ([23]; Examples I.4 \& I.5). For reduced 
rngs $Z C_{2}$ implies $Z C_{n}$ for all $n \geq 2 \quad$ ([23]; Theorem I.3).

3) The notion of reversibility for rings is weaker than that of symmetry. Naturally, if a ring is symmetric, then for any pair $a, b \in A, a b=0$ then $1 a b=0 \Rightarrow 1 b a=b a=0$.

Examples 3.4.7. 1) Fields, integral domains, division rings, domains and zero-rngs are symmetric.

2) Strictly upper triangular matrix rng $\operatorname{SUTM}_{3}(A)$ over any $\operatorname{rng} A$ is symmetric. But it is not reversible. Indeed, if we let $A=\mathrm{Z}$, then $E_{12} E_{23}=E_{13}$ while $E_{23} E_{12}=0$.

3) In Example 3.4.2 (6) in $Z_{2}\left(Q_{8}\right)$, if we set $a=1+z_{i}, b=1+z_{j}$ and $c=1+z_{i}+z_{j}+z_{k}$, then $a b c \neq 0$ but $b a c=0$. This shows that $\mathbf{Z}_{2}\left(Q_{8}\right)$ is not symmetric.

Remark 3.4.8. Notice that, in the rng, with $a b c=0 \Leftrightarrow a c b=0$, there is no guaranty that $b a c=0$. For instance, consider the rng $V$ of Example 3.1.5 (1). Let $c=a+b$. One can easily see that $a b c=0$ but $c a b \neq 0$.

This observation and Remark 3.4.6 (2) suggest a split definition for a symmetric ring. Thus we define:

Definitions 3.4.9. [16] A rng $A$ is called right symmetric if for any triple $a, b, c \in A, a b c=0 \Rightarrow a c b=0$ and left symmetric if $a b c=0 \Rightarrow b a c=0$. If a rng is both left and right symmetric, it is a symmetric rng.

Examples 3.4.10. 1) The rng $V=V_{2}$ as constructed in Example 3.1.5 (1) or in Remarks 3.4.8, is right symmetric but not left symmetric. Obviously, the opposite rng $\left(V_{2}\right)^{\mathrm{op}}$ is left symmetric but not right symmetric.

2) Consider another example which is an extension of above example. Let

$$
V_{3}=\left\{\langle a, b, c\rangle: a^{2}=a b=a c=a ; b^{2}=b a=b c=b ; c^{2}=c a=c b=c\right\}
$$

be with three generators and of characteristic 2. Minor computations show that $V_{3}$ is right symmetric but not left symmetric. On the other hand its opposite rng $\left(V_{3}\right)^{\text {op }}$ is left symmetric and not right symmetric.

3) By iteration, $V_{n}$ and $\left(V_{n}\right)^{\text {op }}$ rngs can be constructed which are right and left symmetric rngs, respectively, but they are not symmetric. For details see [16], where these are termed as generalized Klein- 4 rngs or right absorbing rings. These are zero-divisor rings, and it is proved in [24] that their zero-divisor graphs are precisely the union of a complete graph and a complete bipartite graph.

The following proposition can be followed from [2] [16] and [23].

Proposition 3.4.11. [2] [16] [23] The following are equivalent for any rng $A$.

1) For any $a_{i} \in A$, where $i=1, \cdots, n$, the product $\prod_{i=1}^{n} a_{i}=0 \Leftrightarrow \prod_{i=1}^{n} a_{(i)}=0$, where $\sigma:\{1, \cdots, n\} \rightarrow\{1, \cdots, n\}$ is a permutation.

2) If for any $a, b, c \in A, a b c=0 \Leftrightarrow a c b=0 \Leftrightarrow b a c=0$.

It is also clear that if $A$ is a ring, then

left symmetric $\Leftrightarrow$ symmetric $\Leftrightarrow$ right symmetric.

Proposition 3.4.12. For any rng $A$, the following hold. 
1) Every reduced rng is left and right symmetric, hence it is symmetric.

2) Every left (or right) symmetric rng is an AI-rng.

3) A left (or right) symmetric and reversible rng is symmetric.

4) Every symmetric ring is reversible.

5) Every symmetric rng is an NC-ring.

\section{Proof:}

1) Let $A$ be a reduced rng. Assume that for some $a, b, c \in A$, $a b c=0$. Because every reduced rng is an AI-rng, so

$$
a b c=a c b a c=0 \Rightarrow(a c b)^{2}=(a c b)(a c b)=0 \Rightarrow a c b=0 .
$$

Similarly, $b a c=0$.

2) Let $A$ be a left symmetric rng. Assume that for some $a, b \in A, a b=0$. Then $\forall r \in A, \quad r a b=0 \Rightarrow a r b=0$. Hence $A$ is an AI-rng. The rest of the proof is similar.

3) Let $A$ be a right symmetric and reversible rng. Assume that some $a, b, c \in A$, $a b c=0$. Then $a c b=0 \Rightarrow b a c=0 \Rightarrow b c a=0 \Rightarrow c a b=0$.

4) This holds because of the multiplicative identity.

5) Assume $A$ is a symmetric rng and that for some exponents $x, y, a^{x}=0$ and $b^{y}=0$. Then $a^{x} b^{y}=0$. Hence by Proposition 3.4.12, any binomial coefficient in the form:

$$
a_{1}^{x} b_{1}^{y} \cdots a_{n}^{x} b_{n}^{y}=0, \text { where } x \leq x_{1}+\cdots+x_{n} ; y \leq y_{1}+\cdots+y_{n} .
$$

\section{Some Commutativity Conditions}

In this section we will demonstrate some applications that lead toward commutativity of various types of rngs. In Subsections $4.2 \& 4.3$ we will deal with only rings.

\subsection{A Commutativity Theorem for Semi-Pmc Rngs}

Following theorem is proved in ([25]; Theorem 2.1). But there are some typo errors in the proof. For the sake of typo correction we sketch here an alternate proof of the theorem. Some outcomes are also listed.

Theorem 4.1.1. ([25]; Theorem 2.1) Let $A$ and $B$ be rngs of a semi-pmc $K(A, B)$ in which $\langle,\rangle_{B}$ is epic. If $A$ is commutative and $B$ is reduced, then $B$ is also commutative.

Proof: Assume that the mc

$$
K(A, B)=\left[A, B, M, N,\langle,\rangle_{A},\langle,\rangle_{B}\right]
$$

is a semi-pmc in which $A$ is commutative, $B$ is reduced, and the Morita map $\langle,\rangle_{B}$ is epic. Thus every element of $B$ is a linear combination of Morita pairings of the form $\langle m, n\rangle_{B}$.

Hence, it suffices to prove that an arbitrary commutator

$$
b=\left[\langle m, n\rangle_{B},\left\langle m^{\prime}, n^{\prime}\right\rangle_{B}\right]=0
$$

Straightforward calculations show that $b^{2}=0$, and as $B$ is reduced, $b=0$. 
Some applications of Theorem 4.1.1 are following.

Corollary 4.1.2. Let $A$ and $B$ be rngs of a semi-pmc $K(A, B)$ in which $\langle,\rangle_{B}$ is epic.

1) If $A$ is commutative and $B$ is a domain, then $B$ is also commutative.

2) If $A$ is commutative and $B$ is a division ring, then $B$ becomes a field.

3) If $A$ is commutative and $B$ is an $S R$ rng, then $B$ is also commutative.

4) If $A$ is a nonsingular rng and $N(A) \subseteq$ Cent $(A)$, then $B$ is also commutative.

Proof: All proves are straightforward.

Example 4.1.3. (A semiprime ring which is not reduced)

Let $K(A, B)=\left[A, M, N, B,\langle,\rangle_{A},\langle,\rangle_{B}\right]$ be an mc of rngs and let its Morita rng be denoted by $R=\left[\begin{array}{ll}A & N \\ M & B\end{array}\right]$.

Clearly, if both $A$ and $B$ are rings, then $R$ is a ring. It is proved in ([6]; Theorem 2.1) that from an mc $K(A, B)$ of rings one can always get a semi-pmc and a pmc. In particular, if $\langle,\rangle_{B}$ is epic, then $K(R, B)$ is a pmc. Hence, in this case $B$ and $R$ are Morita similar. Let $K(A, B)$ be a semi-pmc of rings (with $1 \neq 0$ ) in which $\langle,\rangle_{B}$ is epic. It is proved in ([25]; Corollary 2.5) that, if $A$ is commutative and $B$ is reduced, then the Morita ring $R$ is semiprime but not reduced.

\subsection{Right Goldi $C_{k}$-Rings}

Definitions 4.2.1. A ring $\mathrm{A}$ is said to be a $C_{2}$-ring as introduced by Chuang and Lin in1989 in [26], in case for every pair $x, y \in A$, there exist integers $m=m(x, y)$ and $n=n(x, y)$ such that $\left[x^{m}, y^{n}\right]_{k}=0$, where $[x, y]_{k}$ is the $k$ th-commutator defined by $[x, y]_{k}=\left[[x, y]_{k-1}, y\right]$ where $[x, y]_{1}=[x, y]$.

A ring is right Goldie in case it has finite right uniform dimension and satisfies acc on right annihilators.

Lemma 4.2.2: ([26]; Theorem 1) Every reduced $C_{\mathrm{k}}$-ring is commutative.

We sketch here an alternate prove of the following.

Theorem 4.2.3. ([27]; Theorem) A semiprime right Goldie $C_{\mathrm{k}}$-ring with symmetric right quotient is commutative.

Proof: In Proposition 3.4.12 we have proved that every reduced rng is symmetric. Now we prove that the converse also holds for vNR rings. Let $a \in N(A)$ be a non-zero element of some index $n$. Since $A$ is vNR, for some $x \in A$,

$$
a=\operatorname{axa}=(a x)^{n-1} a .
$$

But $A$ is symmetric and $a^{n}=0$, which implies that

$$
a=a^{n} x^{n-1}=0 \text {. }
$$

Hence $A$ is reduced.

The famous Goldie's Theorem states that a $\operatorname{ring} A$ is semiprime right Goldie iff $A$ has a right quotient ring $B$ which is semisimple Artinian ([28]; Theorem 2.3.6). But a semisimple Artinian ring is vNR ([29]; Theorem 1.7). Since $B$ is 
symmetric and now vNR, $B$ is reduced. This means that $A$ is reduced. Since $A$ is a $C_{k}$-ring, by the Lemma $4.1 .2, A$ is commutative.

Proposition 4.2.4. If $A$ is a $v N R$ ring, then

1) $A$ is reduced if and only if $A$ is abelian.

2) $A$ is reduced if and only if $A$ is NCI.

3) $A$ is reduced if and only if $A$ is near- $A I$.

Proof: 1) Let $A$ be reduced. Let $e \in A$ be an idempotent. Assume that $r \in A$. Then $e(1-e)=0$. Because a reduced ring is an AI-ring, $\operatorname{er}(1-e)=0 \Rightarrow e r=$ ere . Similarly one can prove that $r e=e r e$. Hence $e r=r e, \forall r \in A$. Conversely, assume that $A$ is $\mathrm{vNR}$ and abelian. Let $a \in N(A)$. Then for some then $a=a(x a)=x a^{2} \Rightarrow A$ is SR. Hence by 2.2.4(2) $A$ is reduced.

2) Follows from ([11]; Proposition 1.4).

3) Follows from ([13]; Proposition 1.6).

\subsection{Armendariz Rings and Generalizations}

Finally, we very briefly review Armendariz rings.

Definitions 4.3.1. In [30] a rng $A$ is called an Armendariz in case

$$
f(x)=a_{0}+a_{1} x+\cdots+a_{m} x^{m}, g(x)=b_{0}+b_{1} x+\cdots+b_{n} x^{n} \in A[x],
$$

such that

$$
f(x) g(x)=0 \Rightarrow a_{i} b_{j}=0, \forall i=1, \cdots, m ; j=1, \cdots, n .
$$

$A$ is called weak Armendariz in [31] in case

$$
f(x)=a_{0}+a_{1} x, g(x)=b_{0}+b_{1} x \in A[x],
$$

such that

$$
f g=0 \Rightarrow a_{0} b_{0}=a_{0} b_{1}=a_{1} b_{0}=a_{1} b_{1}=0 .
$$

Proposition 4.3.2 For rings following implications hold.

Reduce $\Rightarrow$ Armendariz $\Rightarrow$ weak Armendariz $\Rightarrow$ abelian.

Proof: For: Reduce rings $\Rightarrow$ Armendariz rings see ([13]; Lemma 1.1).

Armendariz rings $\Rightarrow$ weak Armendariz rings holds by definitions.

For: weak Armendariz rings $\Rightarrow$ abelian rings also see ([13]; Lemma 1.1).

Example 4.3.3. Armendariz $\nRightarrow$ Reduced: It is clear from above that every reduced rng is Armendariz but the converse is not true in general as it clear from the following example:

For any reduced ring $A$, the ring of Example 3.3.7.

$$
S=\left\{S_{k}=\left[\begin{array}{ccc}
a_{k} & a_{k 12} & a_{k 13} \\
0 & a_{k} & a_{k 23} \\
0 & 0 & a_{k}
\end{array}\right]: a_{k}, a_{k i j} \in A\right\}
$$

is not reduced. Straightforward calculations show that it is Armendariz.

Example 4.3.4. ([13]; Example 1.2) weak Armendariz $\nRightarrow$ Armendariz.

Consider the factor ring:

$$
A=\mathbf{Z}_{3}[x, y] /\left\langle x^{3}, x^{2} y^{2}, y^{3}\right\rangle .
$$


It is proved in ([31]; Example 3.2) that A is weak Armendariz. Now, in the polynomial ring $A[z]$ over $z, \overline{x y}^{2} \neq 0$ but

$$
(\bar{x}+\bar{y} z)^{3}=(\bar{x}+\bar{y} z)\left(\bar{x}^{2}+2 \bar{x} \bar{y} z+\bar{y}^{2} z^{2}\right)=0 .
$$

Hence $A$ is not Armendariz.

Example 4.3.5. (An abelian ring which is not weak Armendariz) Let $A$ be an abelian ring. Then

$$
R=\left\{\left[\begin{array}{llll}
a & b & c & d \\
0 & a & f & g \\
0 & 0 & a & k \\
0 & 0 & 0 & a
\end{array}\right]: a, b, c, d, f, g, k \in A\right\}
$$

is also abelian (see details in [21]). Then consider the polynomials:

$$
\begin{aligned}
& f(x)=E_{12}+\left(E_{12}-E_{13}\right) x \in R[x] \\
& g(x)=E_{34}+\left(E_{24}+E_{34}\right) x \in R[x]
\end{aligned}
$$

Then $f(x) g(x)=0$ but $E_{12}\left(E_{24}+E_{34}\right) \neq 0$.

Hence $R$ is not weak Armendariz.

Now we give an extended list of equivalent von Neumann regular rings. For proof we refer to ([27]; Lemma). All these rings are NC-rings.

Theorem 4.3.6. Let $A$ be a $v N R$ ring. Then the following are equivalent.

1) $A$ is reduced;

2) $A$ is abelian;

3) $A$ is left (or right) duo;

4) $A$ is left (or right) symmetric,

5) $A$ is reversible;

6) $A$ is 2-primal;

7) $A$ is PS I;

8) $A$ is $N I$;

9) $A$ is $\mathrm{NCI}$

10) $A$ is $A I$;

11) $A$ is quasi-AI;

12) $A$ is near- $A I$;

13) $A$ is Armendariz;

14) $A$ is weak Armendariz,

15) $A$ is a subdirect product of division ring,

16) If $a, a^{\prime}, a^{\prime \prime} \in A$, such that $a a^{\prime \prime}=0=a^{\prime n}$ with $n \geq 1$, then $a a^{\prime} a^{\prime \prime}=0$;

17) If $a, a^{\prime}, a^{\prime \prime} \in A$, such that $a a^{\prime \prime}=0=a^{\prime 2}$, then $a a^{\prime} a^{\prime \prime}=0$.

Remark: For vNR rings above results can also be followed from [9] [11] [13] [14] [15] [32].

The consequences of the Theorems 4.2.3 and 4.3.6 are the following.

Corollary 4.2.7. $A C_{k}-v N R$ ring is commutative if any one of the properties (1)-(17) of Theorem 4.3 .6 is satisfied.

Corollary 4.2.8. A semiprime right Goldie $C_{k}$-ring is commutative if its clas- 
sical ring of quotient satisfies any one of the properties (1)-(17) listed in Theorem 4.3.6.

\section{Conclusion}

This expository work deals with the rings in which sum of two nilpotent elements is nilpotent. All commutative rings have this property, so we have termed them near commutative, or in short, NC-rings. In general, we have considered rings not necessarily be with one. In this work we have picked very common classes of rings which can be subsumed under NC-rings. In any future work more classes of rings can be studied and compared with NC-rings.

\section{Acknowledgements}

This project was funded by the Deanship of Scientific Research (DSR) at King Abdulaziz University, Jeddah, under grant no. G-3-80-30. The authors, therefore, acknowledge with thanks DSR for technical and financial support.

\section{Conflicts of Interest}

The authors declare no conflicts of interest regarding the publication of this paper.

\section{References}

[1] Jacobson, N. (1980) Basic Algebra-II. W. H. Freeman \& Company, San Francisco.

[2] Lambek, J. (1971) On the Representation of Modules by Sheaves of Factor Modules. Canadian Mathematical Bulletin, 14, 359-368. https://doi.org/10.4153/CMB-1971-065-1

[3] Lam; T.Y. (1999) Lectures on Modules and Rings. Graduate Texts in Math., Vol. 189, Springer-Verlag, New-York. https://doi.org/10.1007/978-1-4612-0525-8

[4] Morita, K. (1958) Duality for Modules and Its Applications to the Theory of Rings with Minimum Conditions. Science Reports of the Tokyo Kyoiku Daigaku, 6A, 83-142.

[5] Nauman, S.K. (1994) Intersecting Subcategories of Static Modules, Stable Clifford Theory and Colocalization-Localization. Journal of Algebra, 170, 400-421. https://doi.org/10.1006/jabr.1994.1344

[6] Nauman, S.K. (2004) Morita Similar Matrix Rings and Their Grothendieck Groups. The Alig. Bull. Math, 23, 49-60.

[7] Al-Kenani, A.N. and Nauman, S.K. (2008) A Short Construction of Morita Similar Matrix Rings. JP Journal of Algebra, Number Theory, and Applications, 11, 203-207.

[8] Birkenmeier, G.F., Heatherly, H.E. and Lee, E.K. (1993) Completely Prime Ideals and Associated Radicals, Ring Theory (Granville, $\mathrm{OH}$ 1992). World Scientific Publisher, River Edge, NJ, 102-129.

[9] Marks, G. (2003) A Taxonomy of 2-Primal Rings. Journal of Algebra, 266, 494-520. https://doi.org/10.1016/S0021-8693(03)00301-6

[10] Marks, G. (2001) On 2-Primal Ore Extensions. Communications in Algebra, 29, 2113-2123. https://doi.org/10.1081/AGB-100002173 
[11] Hwang, S.U., Jeon, Y.C. and Park, K.G. (2007) On NCI Rings. Bulletin of the Korean Mathematical Society, 44, 215-223. https://doi.org/10.4134/BKMS.2007.44.2.215

[12] Bell, H.E. (1970) Near Rings in Which Each Element Is a Power of Itself. Bulletin of the Australian Mathematical Society, 2, 363-368. https://doi.org/10.1017/S0004972700042052

[13] Ham, K., Jeon, Y., Kang, J., Kim, N., Lee, W., Lee, Y., Ryu, S. and Yang, H. (2008) IFP Rings and Near-IFP Rings. Journal of the Korean Mathematical Society, 45, 727-740. https://doi.org/10.4134/JKMS.2008.45.3.727

[14] Jeon, Y.C., Kim, H.K., Lee, Y. and Yoon, J.S. (2009) On Weak Armendariz Rings. Bulletin of the Korean Mathematical Society, 46, 135-136. https://doi.org/10.4134/BKMS.2009.46.1.135

[15] Marks, G. (2002) Reversible and Symmetric Rings. Journal of Pure and Applied Algebra, 174, 311-318. https://doi.org/10.1016/S0022-4049(02)00070-1

[16] Nauman, S.K. and Shafee, B.M. (2014) On Extensions of Right Symmetric Rings without Identity. Advances in Pure Mathematics, 4, 665-673.

[17] Shin, G. (1973) Prime Ideals and Sheaf Representations of a Pseudo Symmetric Ring. Transactions of the American Mathematical Society, 184, 43-60. https://doi.org/10.1090/S0002-9947-1973-0338058-9

[18] Huh, C., Lee, Y. and Smoktunowicz, A. (2002) Armendariz Rings and Semicommutative Rings. Communications in Algebra, 30, 751-761. https://doi.org/10.1081/AGB-120013179

[19] Kim, N.K. and Lee, Y. (2003) Extensions of Reversible Rings. Journal of Pure and Applied Algebra, 185, 207-223. https://doi.org/10.1016/S0022-4049(03)00109-9

[20] Habeb, J.M. (1990) A Note on Zero Commutative and Duo Rings. Mathematical Journal of Okayama University, 32, 73-76.

[21] Huh, C., Kim, H.K. and Lee, Y. (2002) Rings and Generalized Rings. Journal of Pure and Applied Algebra, 167, 37-52. https://doi.org/10.1016/S0022-4049(01)00149-9

[22] Cohn, P.M. (1999) Reversible Rings. Bulletin of the London Mathematical Society, 31, 641-648. https://doi.org/10.1112/S0024609399006116

[23] Anderson, D.D. and Camillo, V. (1999) Semigroups and Rings Whose Zero Products Commute. Communications in Algebra, 27, 2847-2852. https://doi.org/10.1080/00927879908826596

[24] Shafee, B.M. and Nauman, S.K. (2015) A Class of Zero Divisor Rings in Which Every Graph Is Precisely the Union of a Complete Graph and a Complete Bipartite Graph. Open Mathematics, 13, 528-536. https://doi.org/10.1515/math-2015-0050

[25] Muthana, N.M. and Nauman, S.K. (2008) Reduced Rings, Morita Contexts and Derivations. East-West Journal of Mathematics, 10, 179-186.

[26] Chuang, L. and Lin, J.S. (1989) On Conjecture of Herstein. Journal of Algebra, 126, 119-138. https://doi.org/10.1016/0021-8693(89)90322-0

[27] Muthana, N.M. and Nauman, S.K. (2012) On Commutativity of Semiprime Right Goldi $\mathrm{C}_{\mathrm{k}}$-Rings. Advances in Pure Mathematics, 2, 217-219.

[28] McConnell, J.C. and Robson, R. (2001) Noncommutative Noetherian Rings. https://doi.org/10.1090/gsm/030

[29] Goodearl, K.R. (1979) von Neumann Regular Rings. Monographs and Studies in Math. 4 Pitman.

[30] Rege, M.B. and Chhawchharia, S. (1997) Armendariz Rings. Proceedings of the Ja- 
pan Academy, Ser. A, Mathematical Sciences, 73, 14-17.

[31] Lee, T.-K. and Wong, T.-L. (2003) On Armendariz Rings. Houston Journal of Mathematics, 29, 583-593.

[32] Kim, H.K., Kim, N.K., Jeong, M.S., Lee, Y., Ryu, S.J. and Yeo, D.E. (2009) On Conditions Provided by Nil Radicals. Journal of the Korean Mathematical Society, 46, 1027-1040. https://doi.org/10.4134/JKMS.2009.46.5.1027 